\title{
BMJ Open Cohort profile: Korean Urban Rural Elderly (KURE) study, a prospective cohort on ageing and health in Korea
}

\author{
Namki Hong, ${ }^{1}$ Kwang-Joon Kim, ${ }^{2}$ Su Jin Lee, ${ }^{3}$ Chang Oh Kim (D) , ${ }^{2}$ \\ Hyeon Chang Kim, ${ }^{3}$ Yumie Rhee, ${ }^{1}$ Yoosik Youm, ${ }^{4}$ Jin-Young Choi, ${ }^{5}$ \\ Hyun-Young Park ${ }^{6}$
}

To cite: Hong N, Kim K-J, Lee SJ, et al. Cohort profile: Korean Urban Rural Elderly (KURE) study, a prospective cohort on ageing and health in Korea. BMJ Open 2019;9:e031018. doi:10.1136/ bmjopen-2019-031018

- Prepublication history and additional material for this paper are available online. To view these files, please visit the journal online (http://dx.doi. org/10.1136/bmjopen-2019031018).

$\mathrm{NH}$ and K-JK contributed equally.

Received 11 April 2019 Revised 04 September 2019 Accepted 18 September 2019

Check for updates

(C) Author(s) (or their employer(s)) 2019. Re-use permitted under CC BY-NC. No commercial re-use. See rights and permissions. Published by BMJ.

For numbered affiliations see end of article.

Correspondence to

Dr Chang Oh Kim;

cokim@yuhs.ac

\section{ABSTRACT}

Purpose The Korean Urban Rural Elderly (KURE) cohort was initiated to study the epidemiologic characteristics, physical performance, laboratory and imaging biomarkers and incidence of age-related diseases in an elderly population with respect to both clinical and social aspects to develop preventive and therapeutic strategies for combatting age-related diseases.

Participants A total of 3517 adults aged 65 or older participated in the cohort at baseline from 2012 to 2015 , recruited from three urban districts and one rural district in Korea. The second-wave follow-up survey is now being conducted at a 4-year interval from baseline (2016-2019; follow-up rate $71.5 \%$ ). The data set included detailed information on anthropometric and socioeconomic factors, functional assessments, image scans (plain radiography, dual-energy X-ray absorptiometry and CT), biospecimens (ie, serum, urine and DNA) and social support networks along with the feasibility of linkage to a national claims database.

Findings to date Mean age of participants at entry was $71.9 \pm 4.6$ years and $67 \%$ were women. From the KURE participants enrolled in baseline recruitment, several studies were published in the fields of cardiometabolic diseases, musculoskeletal health and the association between social support network and diseases in ageing. Future plans Participants will be observed actively and passively every 4-5 years and the first follow-up will be completed in 2020. The KURE data set has strength in comprehensive physical function assessments, quantifiable imaging data sets using CT and detailed information regarding the social support networks of participants from a large community-based elderly Korean population.

\section{INTRODUCTION}

Accelerated ageing of the population poses huge socioeconomic and medical challenges on countries at a global level, not only in terms of proportion of people in the older age group within the whole population but also percentage of oldest old among the older population. ${ }^{1}$ In South Korea, the number of individuals aged $\geq 65$ reached 7 million (14.2\% of the overall population) in 2018, which defines South Korea as an official

\section{Strengths and limitations of this study}

- A total of 3517 adults aged 65 or older participated in the cohort at baseline from 2012 to 2015 , recruited from three urban districts and one rural district in Korea.

- Comprehensive physical function assessments, quantifiable imaging data sets using CT and detailed information regarding the social support networks of participants are unique and strong advantages of this elderly cohort. All data sets and bioresources of the Korean Urban Rural Elderly cohort are managed by the National Biobank of Korea, Korea National Institute of Health, which holds the right to use or share these resources.

- Data linkage with national claims data sources is possible based on unique national identification numbers in the Korean National Health Insurance Service to track health and disease outcomes.

- The second-wave follow-up survey is now being conducted at a 4-year interval from baseline with modest follow-up rate, which was inevitable to an extent owing to the inherent characteristics of an elderly cohort.

- Generalisability of findings to the entire Korean population cannot be guaranteed due to non-randomised sampling design, although this may not critically hinder the analyses of exposure-outcome relationships.

'aged society'. Furthermore, South Korea is expected to progress to a 'super-aged society' within 12 years based on the rapid, on-going trend of ageing, as supported by the world's highest projected life expectancy by $2030 .{ }^{2}$ However, a huge gap still exists between life expectancy and disability-adjusted life expectancy during the same year, which is largely attributed to the increased burden of multiple comorbidities in the elderly population. Cardiovascular diseases, such as coronary heart disease and ischaemic stroke, are the leading cause of years of life lost and years lived with disability, followed by musculoskeletal disease and diabetes. Furthermore, social 
isolation and loneliness pose additional health burdens in older adults, which may have a substantial impact on disability.

Several well-conducted, large cohort studies such as the Health Retirement Study or the Study on global AGEing and adult health (SAGE) studies provided valuable knowledge and insights about human ageing and health issues in older adults in Western population. ${ }^{34}$ However, relative knowledge gap remained for health issues in older adults in South Korea until recently as the representative community-based cohorts, such as The Korean Genome and Epidemiology Study (KoGES) study, mainly focused on the investigation of health issues of middle-aged or younger individuals. ${ }^{5}$ To address the gap, a number of community-based cohort studies have been initiated with the focus on health and ageing in older adults aged 65 or older. The Gwangju Dementia and Mild Cognitive Impairment Study (GDEMCIS) was conducted in 5085 older adults in a single city. ${ }^{6}$ However, only cognitive impairment was focused in the GDEMCIS. The Ansan Geriatric study collected epidemiologic data in 2232 participants in a single rural city, with broader scope on various geriatric diseases than GDEMCIS but this study was conducted based on survey without detailed laboratory or muscle function assessment. ${ }^{7}$ Korean Longitudinal Study on Health and Aging provided well-collected data including physical activity by survey, body composition and laboratory assessments. However, the study had limitations including relatively small sample size with 992 participants recruited in a single city and the lack of information on direct physical function tests and detailed social network size assessments. ${ }^{8}$ The Korean Social Life, Health and Aging Project (KSHAP) cohort assessed broader perspectives of ageing including health behaviours, physical function, blood assays and social network analysis as main focus, whereas the participants were limited to 698 individuals in a single rural district. ${ }^{9}$ Despite the valuable information from all previous cohorts, there is still unmet need in Korea for a more comprehensive, larger sized longitudinal cohort consisted of participants from both urban and rural communities to establish effective strategies for newly emerging health issues related to ageing. Musculoskeletal diseases and sarcopenia in older adults are the major driver of increasing healthcare utilisation in Korea. Changes of muscle function have been highlighted as the core part of musculoskeletal well-being preceding muscle mass changes in older adults. ${ }^{10}$ Previous cohorts in Korea mainly focused on muscle mass measurements for assessing musculoskeletal health but most of studies lacked comprehensive assessment on muscle function. The utilisation of CT in various diseases and individual health assessment (IHA) service using CT in asymptomatic individuals are rapidly expanding in Korea like other developed countries. ${ }^{11}$ However, the feasibility, safety, cost-effectiveness and optimal threshold for 'opportunistic screening' of age-related disorders such as osteoporosis or sarcopenia using CT images obtained for other purpose or during IHA need to be answered in large community-based cohort with CT data.

Despite remarkable economic recovery of Korea, significant rise in income inequality and poverty in older adults in the context of compressed population ageing remains as one of major public policy issues. ${ }^{12}$ The relative and absolute poverty rate reached up to $38.8 \%$ and $38.5 \%$ in older adults in 2006, which are well above the Organisation for Economic Co-operation and Development (OECD) average. Of note, older women have about $10 \%$ higher poverty rate than men, with lower average income of only about a third of men's and higher dependency on public assistance and private transfers from their children. Although few reports proposed the importance of social support network in older women with poverty residing in rural area, further research works are needed to elucidate the role of social support network in vulnerable populations in Korean older adults. ${ }^{13}$

To tackle newly emerging challenges with ageing, the Korean government (Centers for Disease Control and Prevention and the Ministry of Health and Welfare, Korea) initiated a large prospective cohort of community-dwelling older adults aged 65 or older (the Korean Urban Rural Elderly (KURE) cohort). The aim of the KURE cohort was threefold: (1) to provide cross-sectional and longitudinal epidemiologic data of high-impact, modifiable risk factors for cardiovascular, musculoskeletal and metabolic diseases in Korean older adults at earlier stages; (2) to investigate potential indices, including comprehensive assessment on muscle function, physical performance, radiologic and biochemical measurements, which may be useful for preventing and managing future comorbidities for healthy ageing, particularly in relatively healthy individuals at transitioning to octa/nonagenarians; and (3) to assess the impact of social networks on health issues in the older adults, with the focus on the impact of social and geographical differences between rural and urban.

\section{COHORT DESCRIPTION}

\section{Study participants}

To reflect the characteristics of individuals living in urban and rural areas, participants were recruited in four districts (figure 1): three districts located at northwest Seoul (urban area; Eunpyung-gu, Mapo-gu and Seodaemun-gu) and one district located at Incheon (rural area; Ganghwa). Study districts were chosen on the basis of a relatively higher prevalence of the population aged $\geq 65$ years and a low emigration rate to potentially enhance the follow-up rate. Because main purpose of this study was to elucidate the natural course and characteristics of health-related issues in older adults rather than estimating the representative prevalence and incidence of whole Korean population, we chose to construct community-dwelling cohort based on volunteerism to enhance the follow-up rate. We selected three districts at northwest of Seoul city with relatively higher proportion of older 


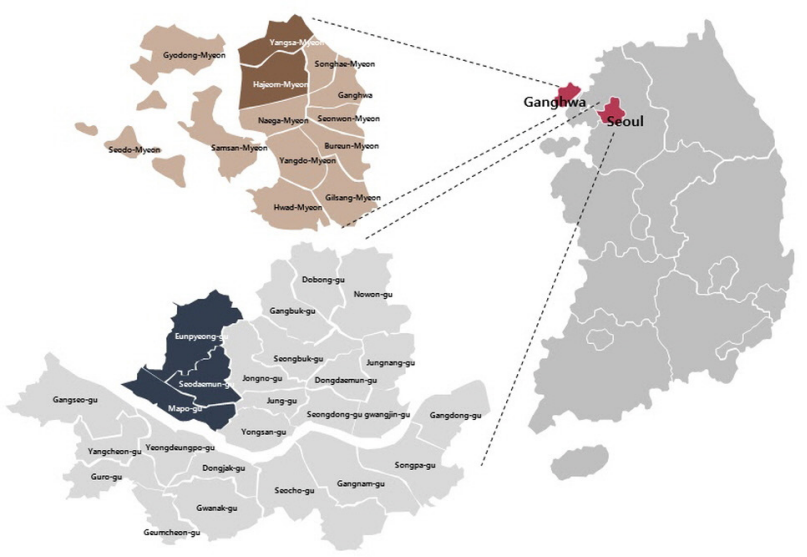

Figure 1 Geographical distribution of study participants in urban and rural areas. Map was drawn by authors by using images sources from open street map.

adults $(10.6 \%-12.2 \%$ in three districts compared with average $9.8 \%$ in Seoul city) to represent urban area with facing new challenges from ageing. Gangwha-gun was selected to represent typical rural area in Korea. More than $42 \%$ of residents in Gangwha-gun engage in agriculture and about $40 \%$ of total area is farmland. Compared with Seoul city, the proportion of older adults is relatively high up to $23.9 \%$, with very low annual emigration rate
(4.9\%), which provides feasibility for long-term follow-up. Participants were recruited using one of following methods: visits to local government health facilities for recruitment, voluntary visit to the study centre after seeing promotional poster for the cohort or acquaintances of study participants. Inclusion criteria were as follows: age $\geq 65$ years; residence in the study area; ability to communicate with the research team; and provision of written informed consent. Those who were below 65 years of age, lived at their current residence for less than 8 months, or planned to relocate from the current residence within 2 years, were excluded from the study (figure 2).

\section{Data collection}

Informed consent was obtained from all participants before the survey and physical examination. Participants underwent trained interviewer-assisted questionnaires regarding socio-demographic status, healthcare utilisation, diet and nutrition, lifestyle, reproductive health, mood disorders and cognitive function (table 1). ${ }^{14}$ In addition to core anthropometric measurements, physical function in the older adults was comprehensively assessed using a brief physical performance battery, including the timed get-up-and-go (TUG) test, chair rise test and hand grip strength measurements, along with a detailed

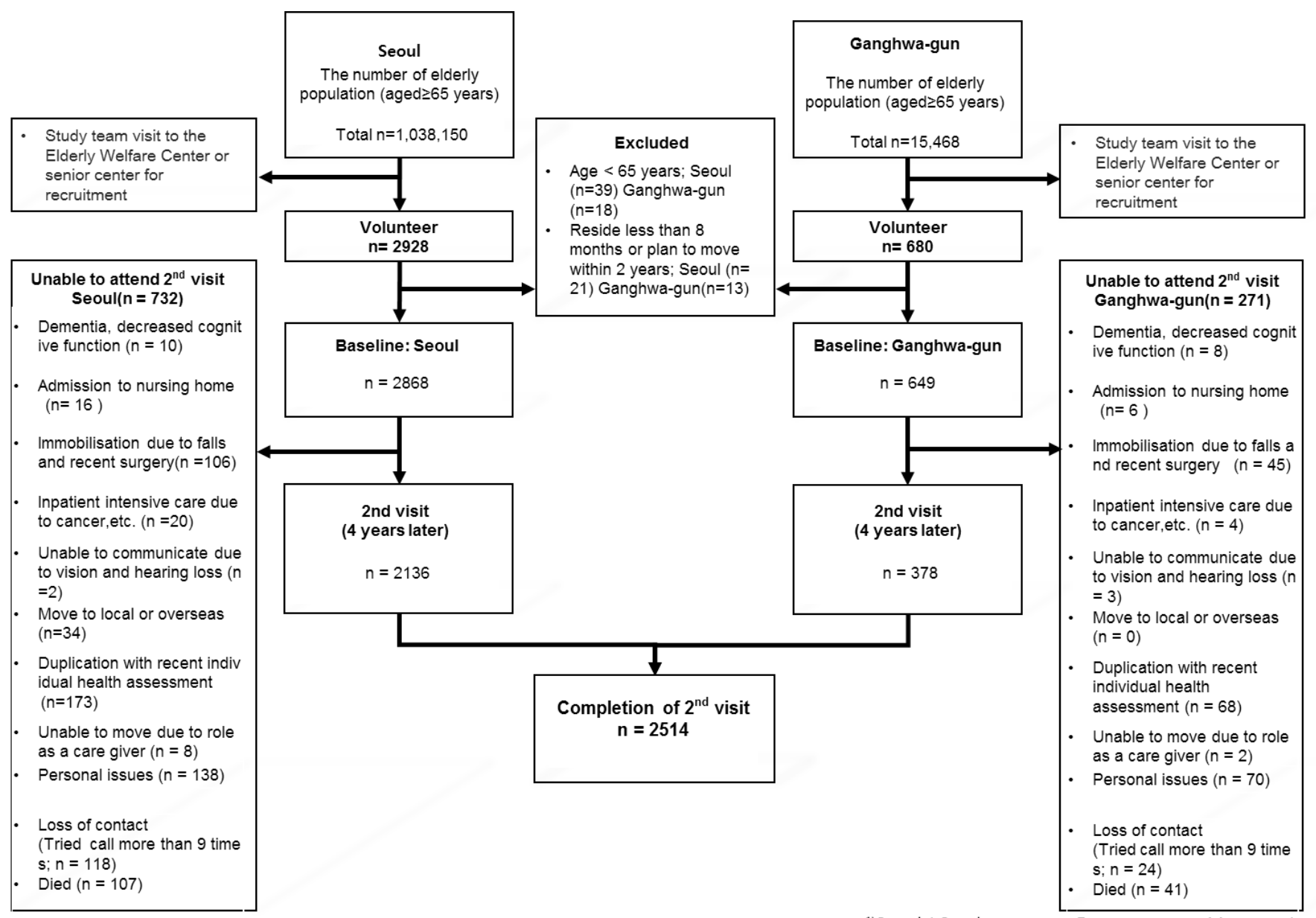

Figure 2 Study flow. 
Table 1 Summary of core variables measured in the Korean Urban Rural Elderly cohort study

\begin{tabular}{|c|c|c|c|}
\hline Category & Measures (instruments) & Baseline & Follow-up \\
\hline $\begin{array}{l}\text { Medical history and } \\
\text { subjective health status }\end{array}$ & $\begin{array}{l}\text { Disease history, disease treatment status, medication } \\
\text { history, family disease history, self-rated health status, } \\
\text { falls and fracture history, lower urinary tract symptoms } \\
\text { (IPSS), daily activity (ADL, IADL), self-rated pain score, } \\
\text { stress scale, reproductive health, frailty (SOF Frailty Index) }\end{array}$ & Yes & $\begin{array}{l}\text { Yes (except education, } \\
\text { marital status, } \\
\text { occupation, stress } \\
\text { scale) }\end{array}$ \\
\hline Health behaviour & $\begin{array}{l}\text { Smoking, alcohol drinking status, sleep disorder, physical } \\
\text { activity (IPAQ-SF), health supplement use }\end{array}$ & Yes & Yes \\
\hline $\begin{array}{l}\text { Anthropometry and } \\
\text { physical examinations }\end{array}$ & $\begin{array}{l}\text { Height, weight, waist, hip, thigh, calf circumference, BIA } \\
\text { for body compositon, blood pressure, pulse rate }\end{array}$ & $\begin{array}{l}\text { Yes (BIA in } \\
\text { subset) }\end{array}$ & Yes \\
\hline Nutritional status & $\begin{array}{l}\text { Nutritional status (Mini-Nutritional Assessment Korean } \\
\text { version) }\end{array}$ & Yes & Yes \\
\hline Mood & Depressive symptoms (GDS-5, GDSSF-K15, SGDS-K) & Yes & Yes \\
\hline Cognitive function & Cognitive function (MMSE-DS Korean) & Yes & Yes \\
\hline Laboratory assessment & $\begin{array}{l}\text { Albumin, alkaline phosphatase, alanine aminotransferase, } \\
\text { aspartate aminotransferase, calcium, blood urea nitrogen, } \\
\text { creatinine, fasting glucose, inorganic phosphorus, protein, } \\
\text { uric acid, total bilirubin, high-sensitivity C-reactive } \\
\text { protein, insulin, total cholesterol, triglyceride, high- } \\
\text { density lipoprotein cholesterol, haemoglobin A1c, white } \\
\text { blood cell count, haemoglobin, platelet count, thyroid- } \\
\text { stimulating hormone, free thyroxine, 25-hydroxyvitamin D, } \\
\text { osteocalcin, troponin T, urinalysis }\end{array}$ & Yes & $\begin{array}{l}\text { Yes (except thyroid- } \\
\text { stimulating hormone } \\
\text { and free thyroxine; } \\
\text { troponin } T \text { in subset) }\end{array}$ \\
\hline
\end{tabular}

Geriatric Depression Scale-Short Form

ADL, Activities of Daily Living; BIA, bioimpedance analysis; DS, dementia screening; GDS-5, Five-item Geriatric Depression Scale; GDSSF-K15, Geriatric Depression Scale Short 15, 15-item Korean version;GFP, ground force plate; IADL, instrumental activities of daily living; IPAQ-SF, International Physical Activity Questionnaire-Short Form; IPSS, International Prostate Symptom Score; MMSE, Mini Mental State Examination; QCT, quantitative computed tomography; SGDS-K, Korean Geriatric Depression Scale-Short Form; SOF, Study of Osteoporotic Fractures; SPPB, Short Physical Performance Battery.

investigation of previous falls and fractures. In a subset of participants, jump power, maximum jump height and velocity were assessed using a ground force plate, which provides a reproducible, physiologic, complex function test with high intensity. Electrocardiography and carotid intima-media thickness measurements were performed to estimate the risk of future cardiovascular events in older adults. Laboratory assessments provided valuable information regarding metabolic status (haemoglobin A1c and fasting glucose, insulin, and thyroid hormone levels), cardiovascular risk (troponin $\mathrm{T}$ and high-sensitivity C-reactive protein levels) and musculoskeletal indices (25-hydroxyvitamin D and osteocalcin levels). Assessment of body composition in older adults plays an important role in the evaluation of sarcopenia and osteoporosis, the two main devastating conditions associated with ageing. At the baseline survey, bone mineral density was measured using dual-energy X-ray absorptiometry (DXA), whereas fat and lean mass was estimated using bioimpedance analysis. In the second follow-up survey, quantitative CT instead of DXA is being used to quantify bone, muscle and fat compartments; this method provides a rich quantifiable data set for the investigation of crosstalk between skeletal muscle, fat content and bone mass 
and geometry according to ageing. The characteristics of social support networks for each participant were assessed using a sophisticated questionnaire developed from the KSHAP, which has been validated in several Korean older adults cohorts. ${ }^{15} 16$ Network size and subjective intimacy were quantified for most participants, which provides a strong and unique advantage of this cohort regarding the analysis of the association between social network characteristics and health status. Biospecimens include serum, plasma, DNA, and urine, which were collected from nearly all participants at the baseline survey and stored in a government-associated central repository.

\section{Patient and public involvement}

Patients and the public were not involved in the design or planning of this study.

\section{Characteristics of study participants by urban and rural area}

Between 2012 and 2015, a total of 3517 participants were enrolled in the KURE cohort. Baseline characteristics of study participants are presented by urban and rural area in table 2. Compared with participants residing in urban area, participants in rural area had older age, lower education level and lower household income. Higher prevalence of current drinking, smoking and underweight (body mass index (BMI) $<18.5 \mathrm{~kg} / \mathrm{m}^{2}$ ) were observed in rural residents. Rural residents had lower physical performance measured by grip strength, TUG tests and chair rise test, which remained robust after adjustment for age and sex using multiple logistic regression (for low grip strength; adjusted OR (aOR) 2.17, 95\% CI 1.73 to 2.73; for low TUG, aOR 1.90, 95\% CI 1.53 to $2.36, \mathrm{p}<0.001$ for all). Prevalence of hypertension was comparable between urban and rural area. However, awareness and control rate of hypertension was significantly lower in rural participants. For diabetes, prevalence was slightly higher in urban area ( 24.8 vs $20.5 \%, \mathrm{p}=0.022$ ), with lower control rate compared with rural participants (34.3 vs $46.2 \%, \mathrm{p}=0.010$ ).

\section{Follow-Up}

The follow-up survey is being performed at 4-year intervals. The second-wave follow-up is now ongoing for participants who enrolled in the cohort in 2014. By the end of 2019, the second-wave follow-up will be completed. The third wave for the 8-year follow-up will be conducted between 2020 and 2023. The KURE team continuously attempted to contact participants on an annual basis by telephone, mail, individual consultation with a health professional after delivery of examination results via telephone, and offline education sessions for a healthy lifestyle to maximise the follow-up rate as possible. The follow-up rate for the second-wave survey was $71.5 \%$ (table 3). Mortality occurred in $4.2 \%$ during follow-up and $24.3 \%$ did not attend to second visit due to immobilisation owing to recent surgery or trauma, admission to a healthcare facility owing to critical illness, admission to a nursing home, inability to communicate owing to decline of cognitive or auditory function and moving to another district (figure 2). Compared with participants who completed follow-up, those who dropped out or deceased had older age, lower physical function, more prevalent current smoking status and higher prevalence of comorbidities including hypertension, diabetes and chronic kidney disease. The proportion of rural residents in dropout (26.7\%) or deceased group (27.7\%) was significantly higher than the average (18.4\%). (table 3) When the reason for dropout was further categorised into health-related issues including nursing home care or hospitalisation, migration out, refusal and loss of contact, rural residents had low migration rate $(0.0 \%)$. (online supplementary table 1) However, the overall follow-up rate in rural area $(58.4 \%)$ was lower than urban $(74.4 \%)$ due to higher rate of dropout due to admission to hospital or nursing home, refusal, loss of contact or mortality, suggesting potential geographical bias between urban and rural area in follow-up.

Among study participants, thyroid cancer $(n=2)$, lung cancer $(n=11)$ and atrial fibrillation $(n=67)$ were incidentally diagnosed by neck ultrasonography performed to measure intima-media thickness, quantitative CT and electrocardiography. All participants with incidental findings were referred to Yonsei university-affiliated hospital (Severance Hospital) for further work-up and treatment. Among them, 40 participants with newly diagnosed atrial fibrillation chose to visit their primary care physician or other clinics.

\section{Findings to date}

Since the publication of the study protocol and completion of baseline recruitment in $2015,{ }^{14}$ several studies have been published or presented at international scientific meetings in various fields including cardiometabolic diseases, musculoskeletal health and social support networks. Given that the second-wave follow-up survey is currently ongoing, longitudinal analyses for each topic have recently been initiated. Therefore, current findings summarised in the following paragraphs are mainly based on investigations using the baseline data set.

\section{Cardiometabolic diseases in ageing}

Given the substantial mortality attributed to cardiovascular events, finding good, sensitive biomarkers that are easily accessible with low cost might help improving strategies for screening and early detection of cardiovascular risk factors in older adults. Published studies from KURE study have concentrated on finding readily available biomarkers for cardiometabolic diseases that are clinically applicable in individuals aged 65 or older, particularly at a subclinical stage. The haemoglobin A1c level was positively associated with carotid intima-media thickness assessed using ultrasonography, even in normoglycemic elderly individuals with haemoglobin A1c levels below $6.5 \% .{ }^{17}$ We found that an elevated uric acid level, a traditional risk factor for gout, was associated with the presence of metabolic syndrome in older adults. ${ }^{18}$ These 
Table 2 Baseline characteristics of study participants in the Korean Urban Rural Elderly cohort by urban and rural area

\begin{tabular}{|c|c|c|c|c|}
\hline & Total & Urban & Rural & \\
\hline Characteristics & $(n=3517)$ & $(n=2870)$ & $(n=647)$ & P value $^{*}$ \\
\hline Women, n (\%) & $2354(66.9)$ & $1987(69.2)$ & $367(56.7)$ & $<0.001$ \\
\hline Age, years & $71.9 \pm 4.6$ & $71.6 \pm 4.5$ & $73.2 \pm 5.0$ & $<0.001$ \\
\hline$<70$ & $1201(34.2)$ & $1038(36.2)$ & $163(25.2)$ & $<0.001$ \\
\hline $70-74$ & 1356 (38.6) & $1123(39.1)$ & $233(36.0)$ & \\
\hline $75-79$ & $723(20.5)$ & $542(18.9)$ & $181(28.0)$ & \\
\hline $80-84$ & $211(6.0)$ & $152(5.3)$ & $59(9.1)$ & \\
\hline 85 or older & $26(0.7)$ & $15(0.5)$ & $11(1.7)$ & \\
\hline \multicolumn{5}{|l|}{ Highest level of education, $n(\%)$} \\
\hline Elementary school & $1871(53.2)$ & $1367(47.7)$ & $504(78.1)$ & $<0.001$ \\
\hline Middle school & $597(17.0)$ & $527(18.4)$ & $70(10.9)$ & \\
\hline High school & $685(19.5)$ & $633(22.0)$ & $52(8.1)$ & \\
\hline College/university or higher & $361(10.3)$ & $342(11.9)$ & $19(2.9)$ & \\
\hline \multicolumn{5}{|c|}{ Household income(US\$/month), n(\%) } \\
\hline Less than 420 & $788(22.5)$ & $540(18.9)$ & 248 (39.2) & $<0.001$ \\
\hline $420-840$ & $819(23.5)$ & 609 (21.3) & 210 (33.2) & \\
\hline $841-1260$ & $630(18.1)$ & $543(19.0)$ & $87(13.7)$ & \\
\hline $1261-1685$ & $319(9.2)$ & $284(10.0)$ & $35(5.5)$ & \\
\hline 1685 or higher & $932(26.7)$ & $879(30.8)$ & $53(8.4)$ & \\
\hline \multicolumn{5}{|l|}{ Physical activity, $n(\%)$} \\
\hline Active & $189(5.4)$ & $154(5.4)$ & $35(5.4)$ & 0.011 \\
\hline Moderate & 1169 (33.2) & $986(34.4)$ & $183(28.3)$ & \\
\hline Inactive & $2157(61.4)$ & $1730(60.2)$ & $429(66.3)$ & \\
\hline \multicolumn{5}{|l|}{ Drinking } \\
\hline Non-drinkers & $2212(62.9)$ & $1830(63.8)$ & $382(59.2)$ & 0.001 \\
\hline Ex-drinkers & $682(19.4)$ & $567(19.8)$ & $115(17.8)$ & \\
\hline Current drinkers & $621(17.7)$ & 473 (16.4) & $148(23.0)$ & \\
\hline \multicolumn{5}{|l|}{ Smoking, n(\%) } \\
\hline Non-smokers & $2585(73.5)$ & $2143(74.7)$ & $442(68.5)$ & 0.003 \\
\hline Ex-smokers & $691(19.7)$ & $547(19.1)$ & $144(22.3)$ & \\
\hline Current smokers & $239(6.8)$ & $180(6.2)$ & $59(9.2)$ & \\
\hline K-MMSE & $24.4 \pm 3.4$ & $24.9 \pm 3.2$ & $22.4 \pm 4.2$ & $<0.001$ \\
\hline $\mathrm{BMI}, \mathrm{kg} / \mathrm{m}^{2}$ & $24.3 \pm 3.0$ & $24.4 \pm 3.0$ & $23.8 \pm 3.3$ & $<0.001$ \\
\hline $\mathrm{BMI}<18.5$ & $91(2.6)$ & $61(2.1)$ & $30(4.6)$ & $<0.001$ \\
\hline BMI 18.5-24.9 & $2057(58.5)$ & $1666(58.1)$ & $391(60.4)$ & \\
\hline BMI 25.0-29.9 & $1236(35.1)$ & $1037(36.1)$ & $199(30.8)$ & \\
\hline $\mathrm{BMI} \geq 30.0$ & $133(3.8)$ & $106(3.7)$ & $27(4.2)$ & \\
\hline \multicolumn{5}{|l|}{ Physical function } \\
\hline Low handgrip strength, $\mathrm{n}(\%) \dagger$ & $531(15.2)$ & $373(13.1)$ & $158(24.8)$ & $<0.001$ \\
\hline Low TUG performance, $\mathrm{n}(\%) \ddagger$ & $591(16.9)$ & $424(14.8)$ & $167(26.2)$ & $<0.001$ \\
\hline Low CRT performance, $n(\%) \S$ & $1026(29.8)$ & $764(27.0)$ & $262(42.9)$ & $<0.001$ \\
\hline \multicolumn{5}{|l|}{ Hypertensionๆ } \\
\hline Prevalence rate, n (\%) & $2204(62.7)$ & $1794(62.5)$ & $410(63.6)$ & 0.652 \\
\hline Awareness rate, $\mathrm{n}(\%)$ & $1918(87.0)$ & $1576(87.9)$ & $342(83.4)$ & 0.018 \\
\hline Treatment rate, $\mathrm{n}(\%)$ & $1855(84.2)$ & $1516(84.5)$ & $339(82.7)$ & 0.369 \\
\hline
\end{tabular}


Table 2 Continued

\begin{tabular}{|c|c|c|c|c|}
\hline & Total & Urban & Rural & \\
\hline Characteristics & $(n=3517)$ & $(n=2870)$ & $(n=647)$ & P value* \\
\hline Control rate, n (\%) & $1410(64.0)$ & 1169 (65.2) & $241(58.8)$ & 0.017 \\
\hline \multicolumn{5}{|l|}{ Diabetes $^{\star \star}$} \\
\hline Prevalence rate, $\mathrm{n}(\%)$ & $843(24.0)$ & $711(24.8)$ & $132(20.5)$ & 0.022 \\
\hline Awareness rate, $\mathrm{n}(\%)$ & $731(86.7)$ & $616(86.6)$ & $115(87.1)$ & 0.999 \\
\hline Control rate, n (\%) & $305(36.2)$ & $244(34.3)$ & $61(46.2)$ & 0.010 \\
\hline
\end{tabular}

${ }^{*} \chi^{2}$ test for categorical variables and one-way analysis of variance for continuous variables.

†Low handgrip strength: $<26 \mathrm{~kg}$ in men; $<18 \mathrm{~kg}$ in women.

łLow TUG performance: $12.6 \mathrm{~s}$ or higher.

$\S$ Low CRT performance: $12 \mathrm{~s}$ or higher for five times of chair rise.

ПHypertension was defined as having one or more following conditions: (1) doctor-diagnosed hypertension; (2) current anti-hypertensive medication use; (3) systolic blood pressure $140 \mathrm{~mm} \mathrm{Hg}$ or higher; or (4) diastolic blood pressure $90 \mathrm{~mm} \mathrm{Hg}$ or higher.

**Diabetes was defined as having one or more following conditions: (1) doctor-diagnosed diabetes; (3) current anti-diabetic medications; (3) fasting glucose $126 \mathrm{mg} / \mathrm{dL}$ or higher; or (4) haemoglobin A1c $6.5 \%$ or higher.

BMI, body mass index; CRT, chair rise test; K-MMSE, Korean version of Mini-Mental State Examination; TUG, timed get-up-and-go;US\$, United States Dollar.

findings suggest that some conventional, widely available blood markers can be used to reflect cardiovascular risks in older adults. Considering the known association between cardiovascular risk factors and vascular dementia, cardiovascular risk factors may affect the initiation and progression of cognitive decline, beyond coronary heart diseases and heart failure. In this context, we found that a low serum 25-hydroxyvitamin D level was associated with higher insulin resistance, cognitive impairment and depressive symptoms. ${ }^{19-21}$ Interestingly, an inverse association between the 25-hydroxyvitamin $\mathrm{D}$ level and insulin resistance was largely explained by regional differences (urban vs rural area). ${ }^{20}$ We also observed that a lower high-density lipoprotein (HDL) cholesterol level, which was known as cardiovascular risk factor, was associated with cognitive impairment, a higher level of dependency in activities of daily living and a poorer chair-rise test performance. $^{22}$ The association of HDL cholesterol with functional state was independent of blood pressure, BMI, history of cardiovascular disease, and health behaviours, suggesting the potential role of cholesterol metabolism in the functional state of older adults beyond a cardiovascular effect. In overall, our findings added evidence for potential, readily available biomarkers for cardiovascular risk, with the extension of previous knowledge regarding association between cardiovascular risk factors and cognitive decline.

\section{Musculoskeletal health in ageing}

Falls and fractures are major adverse health outcomes in older adults, leading to impaired mobility, loss of independence and increased risk of mortality. ${ }^{23}{ }^{24} \mathrm{~A}$ new concept, dysmobility syndrome, was proposed to find individuals at risk of future falls and fractures, beyond merely focusing on bone mineral density testing. Dysmobility syndrome is defined as the presence of three or more of the following factors: a fall in the preceding year, low bone mass, low lean mass, slow gait speed, low grip strength and high fat mass. ${ }^{25}$ However, the association of dysmobility syndrome with musculoskeletal outcomes has never been investigated in Korean older adults. In this cohort, dysmobility syndrome was associated with elevated odds of morphometric vertebral fractures in older Korean adults. ${ }^{26}$ Importantly, dysmobility syndrome improved discrimination for fracture when added to the Fracture Risk Assessment Tool (FRAX) score, a conventional risk assessment tool, suggesting the potential utility of this approach in addition to the current standard of care. Physical function measurements play a central role in assessing the risk of falls and fractures. However, previous methods had some limitations including tester subjectivity, ceiling effect, or testing just simple, isometric action. Jump power measured using a ground force plate has been proposed as a novel, physiologically complex, high-intensity functional marker with good reproducibility and safety. ${ }^{27}$ However, whether this new measurement of physical function is useful in screening individuals at high risk for musculoskeletal impairment has not been investigated yet. In this context, we reported that low jump power was associated with dysmobility syndrome and vertebral fractures, independent of age and covariates. ${ }^{28}$ Along with physical function assessments, we tried to find readily available biomarker for musculoskeletal health. We focused on red distribution width, a marker of cellular senescence, and thyroid hormone status which is known to affect catabolism of muscle and bone. In this cohort, we found independent and additive association of red blood cell distribution width with morphometric vertebral fracture. ${ }^{29} \mathrm{~A}$ low thyroid-stimulating hormone level reflecting subclinical thyrotoxicosis was associated with low bone mass and impaired hip geometry. ${ }^{30}$ In 
Open access

Table 3 Factors associated with dropout and mortality during follow-up

\begin{tabular}{|c|c|c|c|c|c|}
\hline & Total & Completed follow-up & Dropout & Deceased & \\
\hline Characteristics & $(n=3517)$ & $(n=2514)$ & $(n=855)$ & $(n=148)$ & P value* \\
\hline Women, n (\%) & $2354(66.9)$ & $1702(67.7)$ & $603(70.5)$ & $49(33.1)$ & $<0.001$ \\
\hline Rural residents, n (\%) & $647(18.4)$ & $378(15.0)$ & $228(26.7)$ & $41(27.7)$ & $<0.001$ \\
\hline Age, years & $71.9 \pm 4.6$ & $71.3 \pm 4.3$ & $73.1 \pm 4.9$ & $75.5 \pm 5.9$ & $<0.001$ \\
\hline$<70$ & $1201(34.2)$ & $970(38.5)$ & $209(24.4)$ & $22(14.9)$ & $<0.001$ \\
\hline $70-74$ & $1356(38.6)$ & $971(38.6)$ & $333(39.0)$ & $52(35.1)$ & \\
\hline $75-79$ & $723(20.5)$ & $464(18.5)$ & $218(25.5)$ & $41(27.7)$ & \\
\hline $80-84$ & $211(6.0)$ & $105(4.2)$ & $85(9.9)$ & $21(14.2)$ & \\
\hline 85 or older & $26(0.7)$ & $4(0.2)$ & $10(1.2)$ & $12(8.1)$ & \\
\hline \multicolumn{6}{|l|}{ Highest level of education } \\
\hline Elementary school & $1871(53.2)$ & $1255(50.0)$ & $536(62.8)$ & $80(54.4)$ & $<0.001$ \\
\hline Middle school & $597(17.0)$ & $436(17.3)$ & $128(15.0)$ & $33(22.5)$ & \\
\hline High school & $685(19.5)$ & $535(21.3)$ & $132(15.5)$ & $18(12.2)$ & \\
\hline College/university or higher & $361(10.3)$ & $287(11.4)$ & $58(6.7)$ & $16(10.9)$ & \\
\hline \multicolumn{6}{|l|}{ Household income(US\$/month) } \\
\hline Less than 420 & $788(22.6)$ & 507 (20.3) & $233(27.5)$ & $48(33.8)$ & $<0.001$ \\
\hline $420-840$ & $819(23.5)$ & $543(21.8)$ & $235(27.7)$ & $41(28.9)$ & \\
\hline $841-1260$ & $630(18.1)$ & $455(18.2)$ & $159(18.8)$ & $16(11.2)$ & \\
\hline $1261-1685$ & $319(9.1)$ & $238(9.5)$ & $66(7.8)$ & $15(10.6)$ & \\
\hline 1685 or higher & $932(26.7)$ & $755(30.2)$ & $155(18.2)$ & $22(15.5)$ & \\
\hline \multicolumn{6}{|l|}{ Physical activity } \\
\hline Active, n (\%) & $189(5.4)$ & $139(5.6)$ & $47(5.5)$ & $3(2.0)$ & $<0.001$ \\
\hline Moderate, n (\%) & 1169 (33.2) & $793(31.5)$ & $338(39.5)$ & $38(25.7)$ & \\
\hline Inactive, n (\%) & 2159 (61.4) & $1582(62.9)$ & $470(55.0)$ & $107(72.3)$ & \\
\hline \multicolumn{6}{|l|}{ Drinking } \\
\hline Non-drinkers, n (\%) & $2212(62.9)$ & $1543(61.4)$ & $588(68.8)$ & $81(55.1)$ & $<0.001$ \\
\hline Ex-drinkers, n (\%) & $682(19.4)$ & $503(20.0)$ & $151(17.7)$ & $28(19.1)$ & \\
\hline Current drinker, $\mathrm{n}(\%)$ & $621(17.7)$ & $468(18.6)$ & $115(13.5)$ & $38(25.8)$ & \\
\hline \multicolumn{6}{|l|}{ Smoking } \\
\hline Non-smokers, n (\%) & $2585(73.5)$ & $1880(74.7)$ & $641(75.1)$ & $64(43.5)$ & $<0.001$ \\
\hline Ex-smokers, n (\%) & $691(19.7)$ & $492(19.6)$ & $141(16.5)$ & $58(39.2)$ & \\
\hline Current smokers, n (\%) & $239(6.8)$ & $142(5.7)$ & $72(8.4)$ & $25(17.0)$ & \\
\hline K-MMSE & $24.4 \pm 3.6$ & $24.9 \pm 3.2$ & $23.2 \pm 4.0$ & $23.4 \pm 4.1$ & $<0.001$ \\
\hline $\mathrm{BMI}, \mathrm{kg} / \mathrm{m}^{2}$ & $24.3 \pm 3.0$ & $24.2 \pm 2.9$ & $24.4 \pm 3.3$ & $23.7 \pm 3.3$ & 0.034 \\
\hline $\mathrm{BMl}<18.5$ & $91(2.6)$ & $46(1.8)$ & $35(4.1)$ & $10(6.8)$ & $<0.001$ \\
\hline BMI 18.5-24.9 & 2057 (58.5) & $1506(60.0)$ & $462(54.0)$ & $89(60.1)$ & \\
\hline BMI 25.0-29.9 & $1236(35.1)$ & $883(35.1)$ & 307 (35.9) & $46(31.1)$ & \\
\hline $\mathrm{BMI} \geq 30.0$ & $133(3.8)$ & $79(3.1)$ & $51(6.0)$ & $3(2.0)$ & \\
\hline \multicolumn{6}{|l|}{ Physical function } \\
\hline Low handgrip strength† & $536(15.2)$ & $317(12.6)$ & $186(21.7)$ & $33(22.3)$ & $<0.001$ \\
\hline Low TUG performance $\ddagger$ & $591(16.8)$ & $359(14.3)$ & $193(22.6)$ & $39(26.4)$ & $<0.001$ \\
\hline Low CRT performance§ & $1026(29.2)$ & $674(26.8)$ & 292 (34.2) & $60(40.5)$ & $<0.001$ \\
\hline \multicolumn{6}{|l|}{ Comorbidities } \\
\hline Hypertensionף & $2204(62.7)$ & $1546(61.5)$ & $556(65.1)$ & $102(69.4)$ & 0.039 \\
\hline Diabetes $^{\star *}$ & $843(24.0)$ & 568 (22.6) & $232(27.2)$ & $43(29.3)$ & 0.008 \\
\hline
\end{tabular}


Table 3 Continued

\begin{tabular}{|c|c|c|c|c|c|}
\hline & Total & Completed follow-up & Dropout & Deceased & \\
\hline Characteristics & $(n=3517)$ & $(n=2514)$ & $(n=855)$ & $(n=148)$ & P value* \\
\hline Stroke†† & $157(4.5)$ & $104(4.1)$ & $39(4.8)$ & $14(9.5)$ & 0.009 \\
\hline Chronic kidney disease§§ & $270(7.7)$ & $159(6.3)$ & $80(9.4)$ & $31(21.0)$ & $<0.001$ \\
\hline
\end{tabular}

${ }^{*} \chi^{2}$ test for categorical variables and one-way analysis of variance for continuous variables.

†Low handgrip strength: $<26 \mathrm{~kg}$ in men; $<18 \mathrm{~kg}$ in women.

łLow TUG performance: $12.6 \mathrm{~s}$ or higher.

§Low CRT performance: $12 \mathrm{~s}$ or higher for 5 times of chair rise.

१Hypertension was defined as having one or more following conditions: (1) doctor-diagnosed hypertension; (2) current anti-hypertensive medication use; (3) systolic blood pressure $140 \mathrm{~mm} \mathrm{Hg}$ or higher; or (4) diastolic blood pressure $90 \mathrm{~mm} \mathrm{Hg}$ or higher.

**Diabetes was defined as having one or more following conditions: (1) doctor-diagnosed diabetes; (3) current anti-diabetic medications; (3) fasting glucose $126 \mathrm{mg} / \mathrm{dL}$ or higher; or 4) haemoglobin A1c $6.5 \%$ or higher.

$\dagger †$ Stroke was defined as doctor-diagnosed any stroke event in the past.

¥¥Active cancer was defined as doctor-diagnosed cancer receiving active anti-cancer therapy; diagnosed within past 6 months; or recurrent, metastatic or inoperable.

$\S \S C h r o n i c$ kidney disease was defined as doctor-diagnosed chronic kidney disease.

BMI, body mass index; CRT, chair rise test; K-MMSE, Korean version of Mini-Mental State Examination; TUG, timed get-up-and-go;US\$, United States Dollar.

overall, we sought to establish the value of newly emerging concept, dysmobility syndrome and novel biomarkers of musculoskeletal health in this cohort, which need to be continued in further studies.

\section{Social support network and diseases in ageing}

Social support may play a crucial role in diverse health and disease conditions in older adults. ${ }^{31}$ Regarding this concept, we investigated whether social network size and degree of intimacy influence the prevalence of osteoporosis in elderly Korean women. ${ }^{32}$ A large and intimate social support network was associated with a lower prevalence of osteoporosis, whereas a small network size or large but less intimate social network was associated with a higher prevalence of osteoporosis. The association between osteoporosis and poor social support network can be bidirectional that presence of osteoporosis and loss of mobility drive the collapse of social networks whereas the social isolation may hinder early detection and management of osteoporosis and age-related disorders, which need to be validated in further studies. Our findings added evidence for potential influence between health status and social network status in Korea older adults, in line with previous findings.

\section{Strengths and limitations}

The KURE study provides a valuable data set obtained from a large community-based older adults cohort in Korea. Comprehensive physical function assessment, quantifiable imaging data sets using CT and detailed information regarding the social support networks of participants are unique and strong advantages of this elderly cohort. In addition, stored biospecimens prepared using standardised protocols throughout the study will provide opportunities to develop novel biomarkers for age-related diseases. The National Health Insurance Service in Korea is a single-payer, mandatory programme with nearly $100 \%$ coverage for the entire Korean population. Therefore, data linkage with national claims data sources is potentially feasible based on unique national identification numbers to track health and disease outcomes of cohort participants. Taken together, these resources will be useful for capturing a wide range of epidemiologic and clinical characteristics, for exploring the underlying mechanisms, and for developing preventive and therapeutic strategies for ageing-related diseases in older Korean adults.

This study has several limitations. First, follow-up rate is relatively low to modest compared with other similar cohorts. However, decreased response rate is also prevalent phenomenon in recent epidemiologic studies in Korea due to privacy and safety issues in personal information. Limited mobility of older adults with older age, lower physical function, and higher prevalence of comorbidities may also contribute to further decrease in response rate in this population leading to potential loss to follow-up bias, which needs to be considered in future studies using follow-up data in KURE cohort. During the first year of second follow-up study (2016), we tried to perform home visit survey in order to achieve complete follow-up. Among a total of 686 participants followed in 2016, home visit was performed in 126 participants (18.3\%) who could not visit study centre due to various reasons including frailty. Compared with study centre visitors, home visit participants had older age (74 vs 71 years), higher proportion of rural residents (36 vs 16\%) and low household income group (54\% vs $28 \%$ ), lower cognitive function (Korean version of Mini-Mental State Examination score 22 vs 25) and lower physical performance status (low handgrip strength $28 \%$ vs $13 \%$; low chair rise test performance $40 \%$ vs $28 \%$; $<<0.05$ for all) 
(online supplementary table 2). These findings suggest that those who could not visit study centre are more likely to have frailty, requiring home visit to complete the follow-up study. Unfortunately, after 2016, our team could not perform home visit for follow-up due to administrative issues including limited budget and human resources. Therefore, it should be noted that the follow-up study results need to be interpreted on the basis of potential selection bias remaining relatively healthy individuals in the cohort, which warrant thoughtful consideration of researchers in interpreting the results from KURE data set. Although meaningful association can be found, the results should not be extrapolated to all community-dwelling Korean older adults due to inherent bias from follow-up process. As an alternative approach to complement the follow-up, linkage process of KURE data set with national health insurance claim database is now under preparation to obtain indirect follow-up information for health outcomes. Because Korea has universal national health coverage system, almost all data for healthcare use can be obtained via this way along with mortality data and ascertained cause of death records. Another limitation is healthy volunteer bias which may hinder the representativeness of this cohort. Because Korea already had nationwide survey data set and panel studies for descriptive epidemiologic data, it was not the aim of this study to provide the representative sample for older adults in Korea. However, the initial goal of this project proposed by Korea Government was to find novel, high-impact, modifiable health determinants and preventive strategies in cardiovascular and musculoskeletal fields for healthy ageing, particularly for individuals at transition to oldest old groups in rural and urban areas. Because we tried to recruit heterogenous individuals as possible from urban and rural area with mandatory hospital visit, we inevitably relied to volunteerism for this study. Although representativeness of this study could not be guaranteed, we tried to recruit wide spectrum of older adults with various social status and exposure to risk factors. Although the prevalence of hypertension $(62.7 \%)$ and diabetes $(24.0 \%)$ in the KURE cohort was comparable to age-standardised prevalence rates (age, $\geq 65$ years) of hypertension $(64.5 \%)$ and diabetes $(23.4 \%)$ reported in the Korea National Health and Nutrition Examination Survey 2015, we acknowledge that findings from KURE study may not represent the whole Korean older adults, which need to be considered when interpreting results from this cohort. Majority of participants were relatively young with the average age of 71 and only $5 \%$ of participants were octo/nonagenarians, although the proportion of oldest old aged 80 years or older in older adults was relatively lower in Asia (12 \%) than in Europe (20 $\%) .{ }^{1}$ Thus, the data collected at this point may have insufficient power to depict the detailed characteristics of the oldest-old individuals and challenges that they are facing. Given the impact of these oldest old groups expanding fast across high-income countries with major clustering of morbidity and frailty, more attention would be needed for conducting follow-up study for this cohort to capture the culprit factors of healthy ageing in participants transitioning to oldest-old group. At present, relatively short 4-year follow-up data are available for the KURE cohort, although another 4-year follow-up is now being prepared for 2020-2023 to complete 8-year follow-up. Potential geographical bias between urban and rural area in follow-up rate was observed in this cohort. This geographical bias in follow-up rate needs to be considered carefully when interpreting follow-up data of this study.

\section{COLLABORATION}

The KURE cohort offers valuable resources for investigations of human ageing, including comprehensive assessments of musculoskeletal function, social networks, biospecimens and information from various imaging modalities. We are interested in collaboration for ageing and musculoskeletal research based on the available data within KURE. The survey data set will be made available to researchers after quality control process with permission from the Korean Centers for Disease Control and Prevention. All data sets and bioresources of the KURE cohort are managed by the National Biobank of Korea, Korea National Institute of Health, which holds the right to use or share these resources. Biospecimens of the KURE cohort are not available for sharing at this point and further directions regarding sharing archived biologic materials will be announced by the National Biobank of Korea. Researchers who are interested in the KURE cohort are welcome to contact us (email address: jhkwh@nih.go.kr) for future collaboration.

\section{Author affiliations}

${ }^{1}$ Division of Endocrinology, Department of Internal Medicine, Yonsei University College of Medicine, Seoul, South Korea

${ }^{2}$ Division of Geriatrics, Department of Internal Medicine, Yonsei University College of Medicine, Seoul, South Korea

${ }^{3}$ Department of Preventive Medicine, Yonsei University College of Medicine, Seoul, South Korea

${ }^{4}$ Department of Sociology, Yonsei University College of Social Sciences, Seoul, South Korea

${ }^{5}$ Department of Radiology, Yonsei University College of Medicine, Seoul, South Korea ${ }^{6}$ Korea Centers for Disease Control and Prevention, Cheongju, Korea

Acknowledgements We thank all participants, coordinators, staffs and interviewers of the Korean Urban Rural Elderly cohort. The authors appreciate the meticulous laboratory testing procedures performed by Seoul Clinical Laboratories.

Contributors NH and K-JK contributed to the acquisition of the data and draft of manuscript. HCK contributed to the revision of the manuscript, design/supervision of the study and statistical methodology. YR, J-YC and SJL contributed to the revision of the manuscript and design/supervision of the study. YY contributed to the revision of the manuscript with the specific focus on social network methodology. H-YP contributed to the revision of the manuscript, supervision of the study and acquisition of data. COK is a guarantor, who contributed to the design, manuscript drafting and supervision of the study. All authors read and approved the final manuscript.

Funding This work was funded by Research of Korea Centers for Disease Control and Prevention (grant numbers 2013-E63007-01, 2013-E63007-02).

Map disclaimer The depiction of boundaries on the map(s) in this article do not imply the expression of any opinion whatsoever on the part of BMJ (or any member of its group) concerning the legal status of any country, territory, jurisdiction or 
area or of its authorities. The map(s) are provided without any warranty of any kind, either express or implied.

Competing interests None declared.

Patient consent for publication Not required.

Ethics approval This cohort study was approved by the institutional review board of Yonsei University Health System, Severance Hospital (IRB No. 4-2012-0172).

Provenance and peer review Not commissioned; externally peer reviewed.

Data availability statement The survey data collected in KURE cohort are available on reasonable request. The survey dataset will be made available to researchers after quality control process with permission from the Korean Centers for Disease Control and Prevention. All data sets and bioresources of the KURE cohort are managed by the National Biobank of Korea, Korea National Institute of Health, which holds the right to use or share these resources. Biospecimens of the KURE cohort are not available for sharing at this point and further directions regarding sharing archived biologic materials will be announced by the National Biobank of Korea.

Open access This is an open access article distributed in accordance with the Creative Commons Attribution Non Commercial (CC BY-NC 4.0) license, which permits others to distribute, remix, adapt, build upon this work non-commercially, and license their derivative works on different terms, provided the original work is properly cited, appropriate credit is given, any changes made indicated, and the use is non-commercial. See: http://creativecommons.org/licenses/by-nc/4.0/.

ORCID iD

Chang Oh Kim http://orcid.org/0000-0002-0773-5443

\section{REFERENCES}

1 United Nations. World population ageing, 2015.

2 Kontis V, Bennett JE, Mathers CD, et al. Future life expectancy in 35 industrialised countries: projections with a Bayesian model ensemble. Lancet 2017;389:1323-35.

3 Sonnega A, Faul JD, Ofstedal MB, et al. Cohort profile: the health and retirement study (HRS). Int J Epidemiol 2014;43:576-85.

4 Kowal P, Chatterji S, Naidoo N, et al. Data resource profile: the world Health organization study on global ageing and adult health (SAGE). Int J Epidemiol 2012;41:1639-49.

5 Kim Y, Han B-G, KoGES group. Cohort profile: the Korean genome and epidemiology study (KoGES) Consortium. Int J Epidemiol 2017; 46:e20.

6 Lee KS, Cheong HK, BH O, et al. Working criteria of mild cognitive impairment in community: findings from Gwangju dementia and mild cognitive impairment study (GDEMCIS) 2009;1:15-22.

$7 \mathrm{Han} \mathrm{C}$, Jo SA, Kim NH, et al. Study design and methods of the Ansan geriatric study (age study). BMC Neurol 2009;9:10.

8 Park JH, Lim S, Lim JY, et al. An overview of the Korean longitudinal study on health and aging. Psychiatry Investig 2007;4:84-95.

9 Lee J-M, Lee WJ, Kim HC, et al. The Korean social life, health and aging project-health examination cohort. Epidemiol Health 2014;36:e2014003-e03.

10 Goodpaster BH, Park SW, Harris TB, et al. The loss of skeletal muscle strength, mass, and quality in older adults: the health, aging and body composition study. J Gerontol A Biol Sci Med Sci 2006;61:1059-64.

11 Malone J, Del Rosario Perez M, Friberg EG, et al. Justification of CT for Individual Health Assessment of Asymptomatic Persons: A World Health Organization Consultation. J Am Coll Radiol 2016;13:1447-57.

12 Yoon H-S. Korea: balancing economic growth and social protection for older adults. Gerontologist 2013;53:361-8.
13 Lim SJ, Ko Y, Kim C, et al. The death anxiety and depressive symptoms among poor older women in rural areas: the moderating effect of social support. J Korean Acad Community Health Nurs 2017;28:440-9.

14 Lee EY, Kim HC, Rhee Y, et al. The Korean urban rural elderly cohort study: study design and protocol. BMC Geriatr 2014;14:33.

15 Baek J, Hur NW, Kim HC, et al. Sex-Specific effects of social networks on the prevalence, awareness, and control of hypertension among older Korean adults. $J$ Geriatr Cardiol 2016;13:580-6.

16 Youm Y, Laumann EO, Ferraro KF, et al. Social network properties and self-rated health in later life: comparisons from the Korean social life, health, and aging project and the National social life, health and aging project. BMC Geriatr 2014;14:102.

17 Lee SW, Kim HC, Lee Y-ho, et al. Association between HbA1c and carotid atherosclerosis among elderly Koreans with normal fasting glucose. PLoS One 2017;12:e0171761.

18 Choi H, Kim HC, Song BM, et al. Serum uric acid concentration and metabolic syndrome among elderly Koreans: the Korean urban rural elderly (KURE) study. Arch Gerontol Geriatr 2016;64:51-8.

19 Lee EY, Lee SJ, Kim KM, et al. Association of metabolic syndrome and 25-hydroxyvitamin D with cognitive impairment among elderly Koreans. Geriatr Gerontol Int 2017;17:1069-75.

20 Song B, Rhee Y, Kim C, et al. Urban-Rural differences explain the association between serum 25-hydroxyvitamin $D$ level and insulin resistance in Korea. Nutrients 2014;6:5806-18.

21 Song BM, Kim HC, Rhee Y, et al. Association between serum 25-hydroxyvitamin $\mathrm{D}$ concentrations and depressive symptoms in an older Korean population: a cross-sectional study. J Affect Disord 2016;189:357-64.

22 Choi H, Kim HC, Stefani KM, et al. Serum high-density lipoprotein cholesterol concentration and functional state: the Korean urban rural elderly (KURE) study. Arch Gerontol Geriatr 2017;71:115-21.

23 Kado DM, Browner WS, Palermo L, et al. Vertebral fractures and mortality in older women: a prospective study. Arch Intern Med 1999;159:1215-20.

24 Tran T, Bliuc D, van Geel T, et al. Population-Wide impact of Non-Hip Non-Vertebral fractures on mortality. J Bone Miner Res 2017;32:1802-10.

25 Binkley N, Krueger D, Buehring B. What's in a name revisited: should osteoporosis and sarcopenia be considered components of “dysmobility syndrome?". Osteoporos Int 2013;24:2955-9.

26 Hong N, Kim CO, Youm Y, et al. Dysmobility syndrome is associated with prevalent morphometric vertebral fracture in older adults: the Korean urban-rural elderly (KURE) study. Arch Osteoporos 2018;13:86.

27 Buehring B, Krueger D, Binkley N. Jumping mechanography: a potential tool for sarcopenia evaluation in older individuals. J Clin Densitom 2010:13:283-91.

28 Hong N, Kim CO, Youm Y, et al. Low peak jump power is associated with elevated odds of dysmobility syndrome in community-dwelling elderly individuals: the Korean urban rural elderly (KURE) study. Osteoporos Int 2018;29:1427-36.

29 Hong N, Kim CO, Youm Y, et al. Elevated red blood cell distribution width is associated with morphometric vertebral fracture in community-dwelling older adults, independent of anemia, inflammation, and nutritional status: the Korean urban rural elderly (KURE) study. Calcif Tissue Int 2019;104:26-33.

30 Lee SJ, Kim KM, Lee EY, et al. Low normal TSH levels are associated with impaired BMD and hip geometry in the elderly. Aging Dis 2016;7:734-43.

31 Hall A, Wellman B. Social networks and social support. social support and health. San Diego, CA, US: Academic Press 1985:23-41.

32 Lee S, Seo DH, Kim KM, et al. Contingent association between the size of the social support network and osteoporosis among Korean elderly women. PLoS One 2017;12:e0180017. 